\title{
Performance Analysis of IRS-Aided NOMA Communications in the Presence of Imperfect SIC
}

\author{
Van Phu Tuan · Ic-Pyo Hong*
}

\begin{abstract}
The intelligent reflecting surface (IRS) is expected to be a promising technique to achieve a robust spectrum and energy efficiency. This paper investigates the advantages of IRS in enhancing performance of non-orthogonal multiple access (NOMA) communications in the presence of imperfect successive-interference-cancellation (SIC) and phase distortion (PD) caused by a non-ideal IRS. Specifically, average achievable rates (AARs) of the users are the target performance metrics. For performance evaluation, the probabilistic characterizations of signal-to-interference-plus-noise ratios (SINRs) at the users are studied. These results allow for deriving the theoretical formulas for the AAR. Monte Carlo simulations are adopted to verify the accuracy of these theoretical results. The numerical results show the effects of various key system parameters, such as source transmit power, NOMA power allocation (PA) factors, reflecting tile (RTs) allocation, the SIC imperfection factor, and the PD factor, on the AAR that provide useful information for the system's design.
\end{abstract}

Key Words: Achievable Rate Analysis, Imperfect SIC, NOMA, Non-ideal IRS, 5G and Beyond 5G.

\section{INTRODUCTION}

The fifth-generation (5G) network has been being rolled out and has exhibited a remarkable performance, such as a higher data rate, larger network capacity, lower latency, and ubiquitous mobile coverage, compared to its predecessors [1]; however, the exponential growth in data usage demand and the number of wireless devices creates new challenges for the $5 \mathrm{G}$ network as well as the next-generation networks [2]; especially, in the era of Internet of Things (IoT), this problem becomes more critical. Numerous technical efforts have been done for enlarging the network's capability and enhancing the quality of service toward advanced communication applications. For instance, massive multiple-input-multiple-output (MIMO) technology, which uses a large number of antennas to achieve higher coverage and capacity, was introduced. A large millimeter-wave (mmWave) spectrum band is exploited to allow high-speed connectives and high network capacity. Moreover, non-orthogonal multiple access (NOMA), which uses a power-domain multiplexing technique to accommodate several users within an orthogonal resource block, showed a significant improvement in spectrum efficiency [3].

Recently, the intelligent reflecting surface (IRS), which is capable of reflecting electromagnetic waves with a controllable phase-shift and direction, has emerged as a promising technique to realize a smart and programmable wireless environment [4]. The key idea of the IRS is based on special electromagnetic properties on repetitive artificial subwavelength structures [5]. For convenience in manufacturing and deployment, the IRS consists of a large number of independent reflecting tiles (RTs)

Manuscript received November 28, 2020 ; Revised February 19, 2021 ; Accepted April 13, 2021. (ID No. 20201128-194J)

Department of Information and Communication Engineering, Kongju National University, Cheonan, Korea

"Corresponding Author: Ic-Pyo Hong (e-mail: iphong@kongju.ac.kr)

This is an Open-Access article distributed under the terms of the Creative Commons Attribution Non-Commercial License (http://creativecommons.org/licenses/by-nc/4.0) which permits unrestricted non-commercial use, distribution, and reproduction in any medium, provided the original work is properly cited.

(c) Copyright The Korean Institute of Electromagnetic Engineering and Science. 
that are interconnected and controlled by a programmable controller. To verify the advantages of the IRS, the performance of IRS-aided wireless systems has been studied. The works on IRS's physical channel modeling and channel estimation were done in [6-8], allowing for a performance evaluation of IRSaided communication systems. In [9-12], the authors proposed potential applications of the IRS in various communication systems, such as wireless powered networks, secure communications, terahertz communications, cognitive radio systems, and MIMO systems, and showed that these systems gain a significant improvement in performance.

In this paper, we study the advantages of the IRS on enhancing performance of a practical indoor NOMA communication with imperfect successive-interference-cancellation (SIC) and in the presence of phase distortion (PD) caused by a non-ideal IRS. Unlike other approaches, where extravagant state variations are required at the IRS, our approach evaluates average performance metrics, i.e., the average achievable rates (AARs) at users, to significantly reduce IRS's state variations, hence achieving robust power efficiency. First, we adopt a novel channel model proposed for indoor IRS-aided wireless communications in [10] to derive the signal-to-interference-plus-noise ratios (SINRs) for each signal at each user. Next, we study the distribution characterization of relevant performance metrics. Finally, the theoretical AAR formulas can be obtained using the above results. Numerical results are presented for analysis verification and performance evaluation. Moreover, these results point out the effects of system parameters, such as source transmit power, NOMA power allocation (PA) factors, RT allocation, the PD factor, and the SIC imperfection factor, on system performance.

The rest of this paper is organized as follows. The system and channel models are described in Section II. The SINR's distribution and theoretical AAR formulas are studied in Section III. Numerical results and discussions are presented in Section IV. Finally, conclusions are presented in Section V.

Notation: Rice $(\Omega, K)$ denotes the Rice distribution with scale parameter $\Omega$ and shape parameter $K ; \operatorname{CN}\left(0, \delta^{2}\right)$ denotes the complex Gaussian distribution with zero mean and variance $\delta^{2} ; \mathbb{E}\{\cdot\}$ is the expectation of a random variable (RV) $X$.

\section{SYSTEM AND CHANNEL MODELS}

We consider an IRS-aided NOMA communication, as illustrated in Fig. 1, in which a source $S$ communicates with two users, a near user $U_{1}$ and a far user $U_{2}$, with the help of an IRS attached to a wall. All nodes are single-antenna devices. The NOMA technique is used for data transmission for the users. The IRS includes $N$ RTs, $R_{n}, n=1, \ldots, N$, and each RT is a reconfigurable metasurface and can independently direct the signal to a user under the control of a programmable controller.

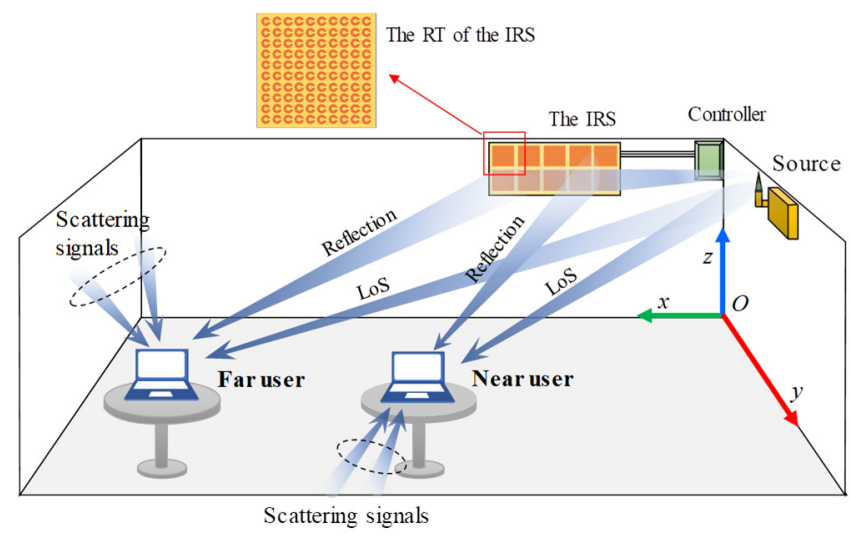

Fig. 1. The system model.

The channel model proposed for indoor IRS-aided wireless communications in $[10,13]$ is adopted to characterize the wireless channels of our system. For instance, the received signal at $U_{m}, m \in 1,2$, comprises of (i) the signals propagating over the line-of-sight (LoS) (or the direct link) and reflecting links, both are the dominant links, and (ii) scattering signals caused by multi-path fading or multiple reflections on the IRS. Let $\mathcal{R}_{m}$ be the set of RTs allocated to $U_{m}, m=\{1,2\}$; hence, the channel between $S$ and $U_{m}$ is expressed as

$$
h_{m}=h_{m}^{\mathrm{LoS}}+h_{m}^{\mathrm{Fad}}+\sum_{R_{n} \in \mathcal{R}_{m}} h_{\mathrm{SR}_{n} \mathrm{U}_{m}}^{\mathrm{Ref}}
$$

where $h_{m}^{\mathrm{LoS}}$ and $h_{\mathrm{SR}_{n} \mathrm{U}_{m}}^{\mathrm{Ref}}$ are channel coefficients for the $\mathrm{LoS}$ link between $S$ and $U_{m}$ and for the reflecting link between $S$ and $U_{m}$ via $R_{n}$, i.e., the $S-R_{n}-U_{m}$ link, respectively, and $h_{m}^{\mathrm{Fad}} \sim C N\left(0, \delta_{\text {Fad }}^{2}\right)$ is the channel coefficient representing the effect of multi-path fading and/or multi-reflection on the RTs.

Let $G_{\mathrm{S}}(\theta, \phi)$ and $G_{\mathrm{U}_{m}}(\theta, \phi)$, respectively denote directivity patterns of the transmit antenna of $S$ and the receive antenna of $U_{m}$ where $\theta$ and $\phi$ are respectively the zenith and azimuth angles. For given locations of $S$ and $U_{m}$ and given antenna's orientations, we can calculate the values for $\theta$ and $\phi$, hence obtaining the values for transmit/receive directivities for wireless links. Let $G_{\mathrm{S}}^{\mathrm{S} \rightarrow \mathrm{X}} \triangleq G_{\mathrm{S}}\left(\theta_{\mathrm{X}}, \phi_{\mathrm{X}}\right)$ be the transmit directivity for the link from $S$ to a given point $X$, and let $G_{\mathrm{U}_{m}}^{\mathrm{Y} \rightarrow \mathrm{U}_{m}} \triangleq G_{\mathrm{U}_{m}}\left(\theta_{\mathrm{Y}}, \phi_{\mathrm{Y}}\right)$ be the receive directivity for the link from a given point $Y$ to $U_{m}$. Using these transmit/receive directivities and the free-space path loss (FSPL) model, we can determine $h_{m}^{\mathrm{LoS}}$ and $h_{\mathrm{SR}_{n} \mathrm{U}_{m}}^{\mathrm{Ref}}$ as

$$
\begin{gathered}
h_{m}^{\mathrm{LoS}}=\sqrt{G_{\mathrm{S}}^{\mathrm{S} \rightarrow \mathrm{U}_{m}} G_{\mathrm{U}_{m}}^{\mathrm{S} \rightarrow \mathrm{U}_{m}}} \frac{\lambda_{0} \mathrm{e}^{-j \varphi_{\mathrm{S}_{m}}}}{4 \pi d_{\mathrm{SU}_{m}}}, \\
h_{\mathrm{SR}_{n} \mathrm{U}_{m}}^{\mathrm{Ref}_{m}}=\sqrt{G_{\mathrm{S}}^{\mathrm{S} \rightarrow \mathrm{R}_{n}} G_{\mathrm{U}_{m}}^{\mathrm{R}_{n} \rightarrow \mathrm{U}_{m}}} \frac{\lambda_{0} \psi_{n} e^{-j \varphi_{\mathrm{SR}_{n} \mathrm{U}_{m}}}}{4 \pi d_{\mathrm{SR}_{n} \mathrm{U}_{m}}},
\end{gathered}
$$


where $\frac{\lambda_{0}}{4 \pi d_{S U_{m}}}$ and $\frac{\lambda_{0}}{4 \pi d_{S R_{n} U_{m}}}$ are the loss factors for the FSPL model with the wavelength $\lambda_{0} ; d_{S U_{m}}$ is the distance between $S$ and $U_{m} ; d_{S R_{n} U_{m}}=d_{S R_{n}}+d_{R_{n} U_{m}}$ is the distance of the $S-$ $R_{n}-U_{m}$ link; $d_{S R_{n}}$ and $d_{R_{n} U_{m}}$ are the distances between $S$ and $R_{n}$ and between $R_{n}$ and $U_{m}$, respectively; $\psi_{\mathrm{R}_{n}}=\beta_{\mathrm{R}_{n}} e^{j \varphi_{\mathrm{R}_{n}}}$ is the reflecting coefficient of $R_{n}$ with $\beta_{R_{n}} \leq 1$ and $\varphi_{\mathrm{R}_{n}} \in[0,2 \pi)$; and $\varphi_{\mathrm{SU}_{m}}=\frac{2 \pi d_{\mathrm{SU}_{m}}}{\lambda_{0}}$ and $\varphi_{\mathrm{SR}_{n} \mathrm{U}_{m}}=\frac{2 \pi d_{\mathrm{SR}_{n} \mathrm{U}_{m}}}{\lambda_{0}}$ are the phase shifts caused by wave propagation over the distances $d_{S U_{m}}$ and $d_{S R_{n} U_{m}}$.

As shown in (1), $h_{m}$ is a Rice distributed RV. Let $h \sim$ $\operatorname{Rice}(\Omega, K)$ be a general Rice distributed RV, then the cumulative distribution function $(\mathrm{CDF})$ of $|h|^{2}$ is expressed as [10]:

$$
F_{0}(x ; K, \Omega)=1-e^{-\mu x} \sum_{k=0}^{\infty} \mathrm{B}_{k}(\mu x)^{k},
$$

where $\mu=\sigma_{\text {Fad }}^{-2}$ and $\mathrm{B}_{k}=\left(1-e^{-K} \sum_{i=0}^{k-1} \frac{K^{i}}{i !}\right) / k$ !

Next, we study the effect of PD caused by non-ideal RTs on the received signal at the users. Regarding the NOMA principle, $S$ transmits a combined signal $x_{S}=\sqrt{\alpha_{1}} x_{1}+\sqrt{\alpha_{1}} x_{2}$ to the users where $x_{1}, \mathrm{E}\left\{\left|x_{1}\right|^{2}\right\}=P_{0}$, and $x_{2}, \mathrm{E}\left\{\left|x_{2}\right|^{2}\right\}=P_{0}$, are respectively the desired signals for $U_{1}$ and $U_{2} ; \alpha_{1}$ and $\alpha_{2}$ are the PA factors of NOMA satisfying $\alpha_{1}+\alpha_{2}=1$. When $x_{\mathrm{s}}$ is reflected from the RTs, the PD caused by the non-ideal RTs make it distorted. The effect of non-ideal hardware on system performance has been widely studied in [14-16] and is characterized as a complex Gaussian noise $\kappa_{\mathrm{R}_{n}}^{\mathrm{PD}} \sim \mathrm{CN}\left(0, \tau_{\mathrm{R}_{n}} P_{0}\right)$ with the PD factor $\tau_{\mathrm{R}_{n}} \ll 1$. Using (1), the received signal for $U_{m}$ in the presence of $\mathrm{PD}$ is given by

$$
y_{m}=h_{m}^{\mathrm{LoS}} x_{s}+h_{m}^{\mathrm{Fad}} x_{s}+\left(\sum_{\mathrm{R}_{n} \in \mathrm{R}_{m}} h_{\mathrm{SR}_{n} \mathrm{U}_{m}}^{\mathrm{Ref}} \hat{x}_{s}\right)+n_{m},
$$

where $n_{m} \sim \operatorname{CN}\left(0, N_{0}\right)$ is the additive white Gaussian noise (AWGN) at the receive antenna, and $\hat{x}_{s} \triangleq x_{s} \sqrt{1-\tau_{R_{n}}}+\kappa_{R_{n}}^{\mathrm{PD}}$ is the distorted signal reflected on $R_{n}$. The factor $\sqrt{1-\tau_{R_{n}}}$ is the power-constraint factor for guaranteeing $\mathbb{E}\left\{\left|\hat{x}_{S}\right|^{2}\right\}=$ $\mathbb{E}\left\{\left|x_{s}\right|^{2}\right\}=P_{0}$, or equivalently, the IRS does not add any power to the reflected signal. We can rewrite (5) as

$$
\begin{gathered}
y_{m}=\underbrace{\left(h_{m}^{\mathrm{LoS}}+h_{m}^{\mathrm{Fad}}+\sum_{\mathrm{R}_{n} \in \mathbf{R}_{m}} \sqrt{1-\tau_{\mathrm{R}_{n}}} h_{\mathrm{SR}_{n} \mathrm{U}_{m}}^{\mathrm{Ref}}\right)}_{\hat{h}_{m}} x_{s} \\
+\underbrace{n_{\mathrm{U}_{m}}+\sum_{\mathrm{R}_{n} \in \mathbf{R}_{m}} h_{\mathrm{SR}_{n} \mathrm{U}_{m}}^{\mathrm{Ref}} \kappa_{\mathrm{R}_{n}}^{\mathrm{PD}}}_{\mathrm{U}_{m}},
\end{gathered}
$$

It is seen from (6) that $\hat{h}_{m} \sim \operatorname{Rice}\left(\hat{\Omega}_{m}, \hat{K}_{m}\right)$ is still a Rice distributed $\mathrm{RV}$, and $\hat{n}_{m} \sim \mathrm{CN}\left(0, N_{0}+\sigma_{\mathrm{PD}}^{2}\right)$ is the total noise including the AWGN and the noise caused by the PD. The values for $\hat{\Omega}_{m}, \hat{K}_{m}$, and $\sigma_{\mathrm{PD}}^{2}$ are calculated as the follows.

The phase shift of each RT, i.e., $\varphi_{\mathrm{R}_{n}}$, plays an important role in system performance. It defines whether the received signals at $U_{m}$ via the dominant links are constructive or destructive. Let us consider the distance-propagation phase shift of the LoS link, i.e, $\varphi_{\mathrm{SU}_{m}}$, as the standard reference in the phase shift at $U_{m}$, the signal-combining phase shift denoted by $\varphi_{R_{n}}^{s c}$, which measures the difference between the distance-propagation phase shift on the $S-R_{n}-U_{m}$ link and $\varphi_{\mathrm{SU}_{m}} . \varphi_{R_{n}}^{S C}$ is calculated as follows [17].

$$
\varphi_{\mathrm{R}_{n}}^{\mathrm{Sc}}=\bmod \left(\varphi_{\mathrm{R}_{n}}+\varphi_{\mathrm{SR}_{n} \mathrm{U}_{m}}-\varphi_{\mathrm{SU}_{m}}, 2 \pi\right) .
$$

Using $\varphi_{R_{n}}^{S c}, \psi_{\mathrm{R}_{n}}, h_{m}^{\mathrm{Fad}}$, and the formulas for channel coefficients $h_{m}^{\mathrm{LOS}}$ and $h_{\mathrm{SR}_{n} \mathrm{U}_{m}}^{\mathrm{Ref}}$ (given in (2) and (3)), we can calculate $\widehat{\Omega}_{m}$ and $\widehat{K}_{m}$ using a similar approach in [10]:

$$
\begin{gathered}
\widehat{\Omega}=\sigma_{F a d}^{2}+\left(\frac{\lambda_{0}}{4 \pi}\right)^{2}\left(\left(\frac{\sqrt{G_{S}^{S \rightarrow U_{m}} G_{U_{m}}^{S \rightarrow U_{m}}}}{d_{S U_{m}}}+\right.\right. \\
\left.\sum_{R_{n} \in \mathcal{R}_{m}} \frac{\sqrt{\left(1-\tau_{R_{n}}\right) G_{S}^{S \rightarrow R_{n}} G_{U_{m}}^{R_{n} \rightarrow U_{m}}} \beta_{R_{n}} \cos \left(\varphi_{R_{n}}^{s c}\right)}{d_{S R_{n} U_{m}}}\right)^{2}+ \\
\left.\left(\sum_{R_{n} \in \mathcal{R}_{m}} \frac{\sqrt{\left(1-\tau_{R_{n}}\right) G_{S}^{S \rightarrow R_{n}} G_{U_{m}}^{R_{n} \rightarrow U_{m}}} \beta_{R_{n}} \sin \left(\varphi_{R_{n}}^{S c}\right)}{d_{S R_{n} U_{m}}}\right)^{2}\right), \\
\widehat{K}_{m}=\widehat{\Omega}_{m} / \sigma_{F a d}^{2} .
\end{gathered}
$$

Then, using the fact that the sum of independent complex Gaussian RVs is a complex Gaussian RV with its variance being the sum of all variances, we can calculate $\sigma_{\mathrm{PD}}^{2}$ as

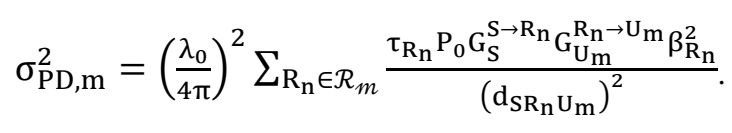

\section{SINR DISTRIBUTION CHARACTERIZATION AND}

\section{Average ACHIEVABLE RATE ANALYSIS}

For convenience in analyzing the SINRs, we rewrite (5) as

$$
y_{m}=\hat{h}_{m}\left(\alpha_{1} x_{1}+\alpha_{2} x_{2}\right)+\hat{n}_{m} .
$$

According to the SIC principle of NOMA, the SINRs for the signal $x_{2}$ at both users, i.e., $U_{m}, m=\{1,2\}$, are given by

$$
Y_{m}=\frac{\gamma_{m}\left|\hat{h}_{m}\right|^{2} \alpha_{2}}{1+\gamma_{m}\left|\hat{h}_{m}\right|^{2} \alpha_{1}}
$$


where $\gamma_{m}=P_{0} /\left(N_{0}+\sigma_{\mathrm{PD}, m}^{2}\right)$, and the SINR for the signal $x_{1}$ at $U_{1}$ is given by

$$
X=\frac{\gamma_{1}\left|\hat{h}_{1}\right|^{2} \alpha_{1}}{1+\gamma_{1}|g|^{2} \alpha_{2}} .
$$

Using Shannon's law, we can calculate the achievable rates (also known as the ergodic capacities) in bits $/ \mathrm{s} / \mathrm{Hz}$ for the signal $x_{2}$ at both users as

$$
R_{2}=\log _{2}(1+Y),
$$

and for the signal $x_{1}$ at $U_{1}$ as

$$
R_{1}=R_{x_{1} \mid U_{1}}=\log _{2}(1+X),
$$

where $Y=\min \left(Y_{1}, Y_{2}\right)$ is the condition for guaranteeing successfully decoding $x_{2}$ at both users (note that $U_{1}$ must decode $x_{2}$ before decoding its information $\left.x_{1}\right)$, and $g \sim \mathrm{CN}\left(0, \lambda_{\text {ImSIC }}\right)$ in (13) represents the residual signal of $x_{2}$ caused by the imperfect SIC receiver at $U_{1}$ with variance $\lambda_{\mathrm{sICIm}}=\zeta \mathrm{E}\left\{\left|h_{\mathrm{SU}_{1}}\right|^{2}\right\}$ and the SIC imperfection factor $\zeta \leq 1$.

Proposition 1. The CDF of $X$ denoted by $F_{X}(t)=\operatorname{Pr}(X<t)$ is approximated as

$$
\begin{aligned}
F_{X}(t) & =1-\rho_{1} e^{\rho_{1} \eta} \sum_{k=0}^{\infty} \mathrm{B}_{k} \\
& \times \frac{t^{k}}{(\eta+t)^{k+1}} \Gamma\left(k+1, \rho_{1}(\eta+t)\right),
\end{aligned}
$$

where $\rho_{m}=\mu /\left(\alpha_{1} \gamma_{m}\right)$, and $\eta=\lambda_{\text {ImSIC }} \alpha_{1} /\left(\mu \alpha_{2}\right)$.

Proof. The approximate CDF of $X$ is calculated as $F_{X}(t)$ $\approx \operatorname{Pr}\left(\left|\hat{h}_{1}\right|^{2}<\left(1+\gamma_{1}|g|^{2} \alpha_{2}\right) t /\left(\gamma_{1} \alpha_{1}\right)\right)$. Using the probability density function (PDF) of the exponential distribution given by $f_{|g|^{2}}(t)=\lambda_{\text {ImSIC }} \exp \left(-\lambda_{\text {ImSIC }} t\right)$ and (4), we obtain $F_{X}(t)$ via solving the following equation.

$$
\begin{aligned}
& F_{X}(t) \\
& =\int_{0}^{+\infty} \lambda_{\text {imSIC }} \mathrm{e}^{-\lambda_{\text {imsIC }} \mathrm{F}_{0}}\left(\frac{1}{\gamma_{1} \alpha_{1}}\left(1+\gamma_{1} \alpha_{2} \mathrm{x}\right) \mathrm{t} ; \widehat{\Omega}_{1}, \widehat{\mathrm{K}}_{1}\right) \mathrm{dx} \\
& =1-\eta \sum_{\mathrm{k}=0}^{\infty} \mathcal{B}_{k}\left(\rho_{1}\right)^{\mathrm{k}+1} \mathrm{e}^{\rho_{1} \eta} \mathrm{t}^{\mathrm{k}} \int_{1}^{+\infty} e^{-\rho_{1}(\eta+t) y} y^{k} \mathrm{dy},
\end{aligned}
$$

Using [18, Eq. (3.381.3)] and some manipulations, we obtain (16). The proof for Proposition 1 is completed. $\square$

Proposition 2. The CDF of $Y$ denoted by $F_{Y}(t)=\operatorname{Pr}(Y<t)$ is equal to one if $t>\alpha_{2} / \alpha_{1}$, and otherwise, it is calculated as

$$
\begin{aligned}
F_{\mathrm{Y}}(t)= & 1-\left(1-F_{0}\left(\frac{t}{\gamma_{1}\left(\alpha_{2}-t \alpha_{1}\right)} ; \widehat{\Omega}_{1}, \widehat{K}_{1}\right)\right) \\
& \times\left(1-F_{0}\left(\frac{t}{\gamma_{2}\left(\alpha_{2}-t \alpha_{1}\right)} ; \widehat{\Omega}_{2}, \widehat{K}_{2}\right)\right)
\end{aligned}
$$

Proof. Since $Y=\min \left(Y_{1}, Y_{2}\right)$, we can rewrite $F_{Y}(t)$ as $F_{Y}(t)=1-\operatorname{Pr}\left(Y_{1}>t\right) \operatorname{Pr}\left(Y_{2}>t\right)$. Then, substituting (4) into it yields (18). The proof for Proposition 2 is completed. $\square$

Using Propositions 1 and 2, we can obtain the theoretical results for AARs as follows.

Proposition 3. The theoretical AAR expressions for the signals $x_{1}$ and $x_{2}$ are respectively given in the equations (19) and (20) shown in the top of the next page, where $C_{k}=\sum_{q=0}^{k} B_{1,9} B_{2, k-q}\left(\gamma_{1}\right)^{-q}\left(\gamma_{2}\right)^{-k+q}$.

$$
\begin{aligned}
& \bar{C}_{1}=\frac{1}{\ln (2)} \eta e^{\eta \rho_{1}} \sum_{k=0}^{\infty} \mathrm{B}_{k} \int_{0}^{\infty} \frac{t^{k}}{(1+t)(\eta+t)^{k+1}} \Gamma\left(k+1, \rho_{1}(\eta+t)\right) d t \\
& \bar{C}_{2}=\frac{1}{\ln (2)} \sum_{k=0}^{\infty} C_{k} \mu^{k} \int_{0}^{\alpha_{2} / \alpha_{1}} \frac{t^{k}}{(1+t)\left(\alpha_{2}-t \alpha_{1}\right)^{k}} e^{-\left(\frac{\mu}{\eta_{1}}+\frac{\mu}{\gamma_{2}}\right) \frac{t}{\left(\alpha_{2}-t \alpha_{1}\right)}} d t
\end{aligned}
$$

Proof. According to [19], the AAR for the signal $x_{m}, m=\{1$, $2\}$, is calculated as

$$
\bar{C}_{m}=\mathrm{E}\left\{R_{m}\right\}=\frac{1}{\ln (2)} \int_{0}^{+\infty} \frac{1-F_{m}(t)}{1+t} d t .
$$

Substituting (16) and (17) into (21) and using some manipulations, we can obtain (19) and (20). The proof for Proposition 3 is completed. $\square$

\section{NUMERICAL RESULTS AND DISCUSSION}

In this section, we present the numerical results for the AAR and discuss the effect of different key parameters on it. The theoretical results are derived for a general system, such as any type of transmit/receive directivity of the device antennas, frequency, wireless indoor environment, and device locations. For the simulation, we chose a specific setup for our system. Without loss of generality, we set the phase shifts of the IRS as $\varphi_{R_{n}}=$ $2 \pi \times \bmod \left(d_{\mathrm{SR}_{n}}+d_{\mathrm{R}_{n} \mathrm{U}_{m}}-d_{\mathrm{SU}_{m}}, \lambda_{0}\right) / \lambda_{0}$ to gain the strongest received signal strengths (RSSs) at the users, $\beta_{R_{n}}=0.9$ and $\tau_{R_{n}}=0.1$ where $n=1, \ldots, 2 N_{\text {col }}$. The coordinate setups are shown in Fig. 2, and the setup of other parameters is listed in Table 1 [20]. The transmit antenna is a half-wavelength dipole with its orientation illustrated in Fig. 2, and its directivity pattern is given in Table 1 . The receive antennas have the same directivity for all directions. The numerical results include the theoretical AARs obtained using (19) and (20), and the simulation results are obtained by evaluating a simulation system using MATLAB. The good match between the theoretical and the simulation results confirms the accuracy of our study.

In Fig. 3, we show the advantage of using the IRS to assist NOMA communication via examining a simple IRS, including 2 RTs. We consider four possible cases denoted by 
TUAN and HONG: PERFORMANCE ANALYSIS OF IRS-AIDED NOMA COMMUNICATIONS IN THE PRESENCE OF IMPERFECT SIC

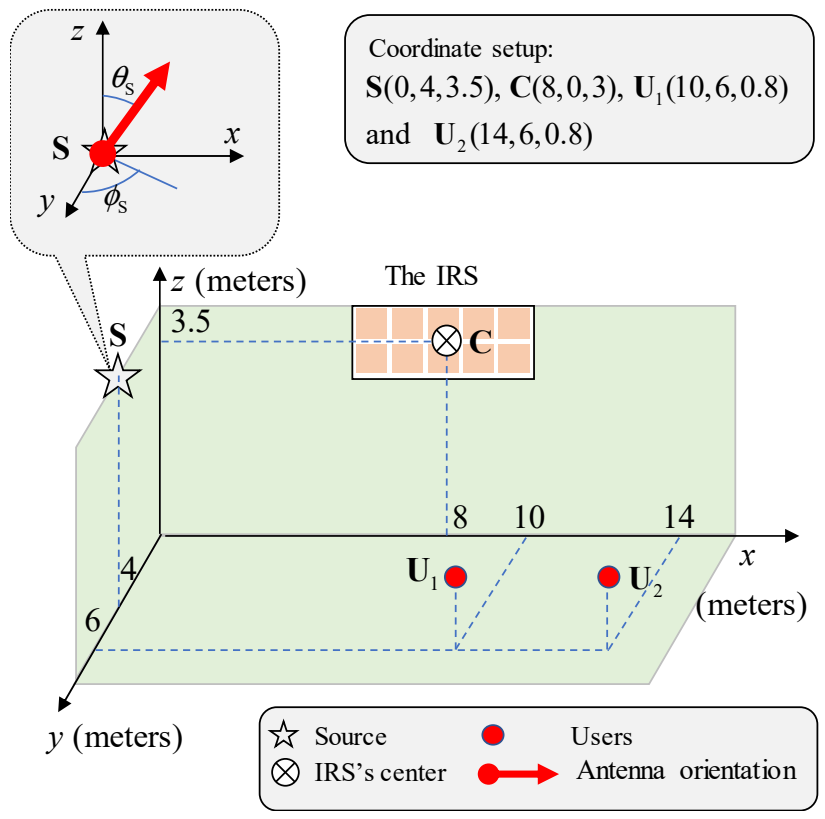

Fig. 2. The coordinate setups for the simulation.
Table 1. Parameter setups for the simulation

\begin{tabular}{lc}
\hline \multicolumn{1}{c}{ Parameter } & Value \\
\hline Frequency $(\mathrm{GHz})$ & $f_{0}=6$ \\
Size & $2 \times N_{\text {col }} \mathrm{RT}$ \\
$\quad$ IRS (row $\times$ column) & $0.5 \times 0.5$ \\
$\quad$ RT $\left(\mathrm{m}^{2}\right)$ & $\tau_{R_{n}}=\tau_{P D}=0.1$ \\
PD factor & $P_{S}=0$ \\
Transmit power $(\mathrm{dBm})$ & $\left\{\sigma_{\text {Fad }}^{2}, N_{0}\right\}=\{-68.7,-70\}$ \\
Fading \& AWGN $(\mathrm{dBm})$ & $\zeta=0.04$ \\
SIC receiver & Orientation: $:\left\{\theta_{\mathrm{S}}, \varphi_{\mathrm{S}}\right\}=\left\{20^{\circ}, 0^{\circ}\right\}$. \\
Tx-antenna setup & $G_{\mathrm{s}}(\hat{\theta}, \hat{\phi})=1.67 \sin (\hat{\theta})^{3}[20$, Eq. $(2-18)]$. \\
\end{tabular}

Note that $\hat{\theta}$ is the angle between the antenna-orientation vector and the vector from $\mathrm{S}$ to the considered point. $\hat{\phi}$ is not important, so we do not consider its value.

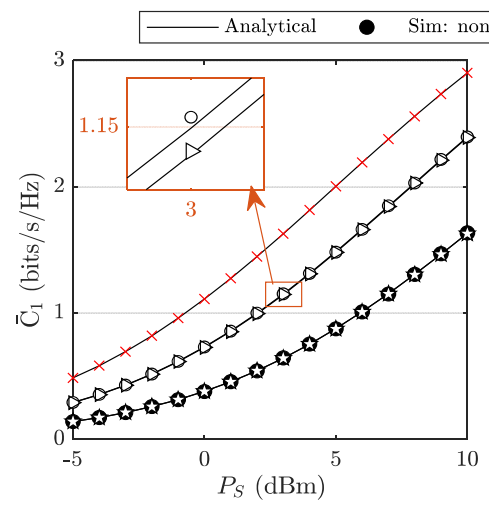

(a)

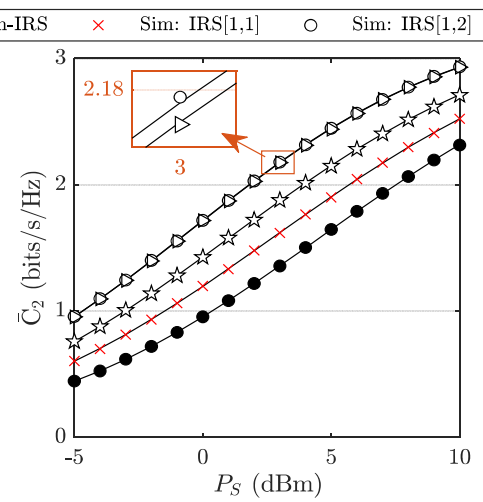

(b)

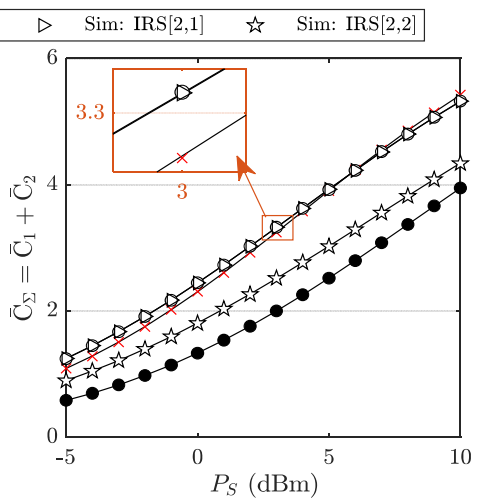

(c)

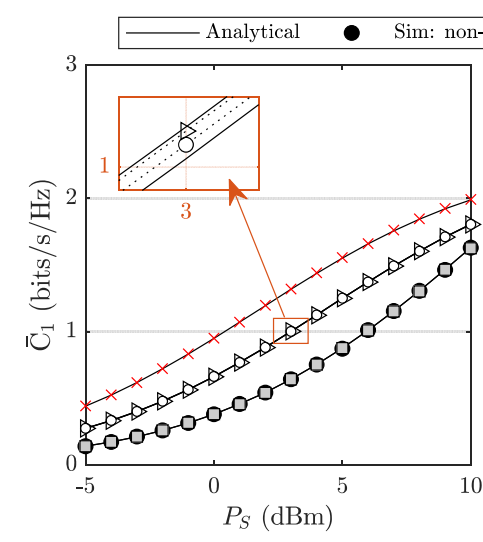

(d)

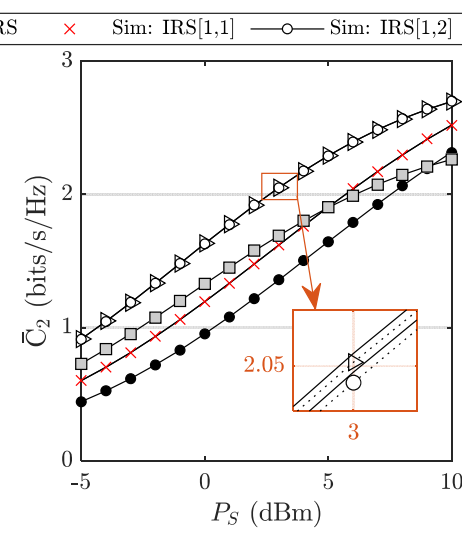

(e)

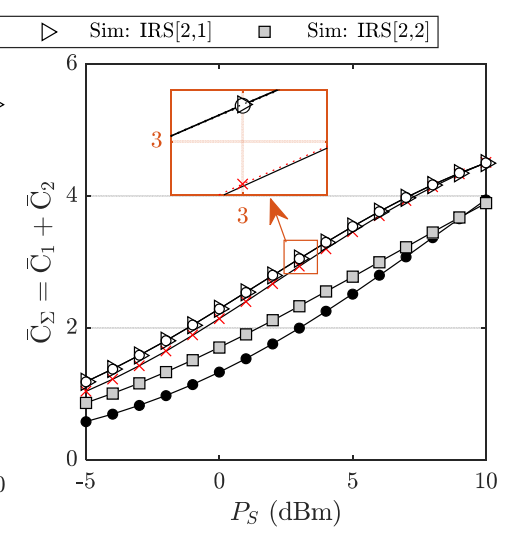

(f)

Fig. 3. The results of the system's capacities for the ideal-RT scenario: (a) AAR for signal $x_{1}$, (b) AAR for signal $x_{2}$, and (c) total AAR. For the non-ideal-RT scenario: (d) AAR for signal $x_{1}$, (e) AAR for signal $x_{2}$, and (f) total AAR.

$\operatorname{IRS}\left[m_{1}, m_{2}\right], m_{1}, m_{2} \in\{1,2\}$, which indicates that the first and the second RTs are allocated to $U_{m_{1}}$ and $U_{m_{2}}$, respectively. The AARs for $x_{1}, x_{2}$ and total AAR, $\bar{C}_{\Sigma}=\bar{C}_{1}+\bar{C}_{2}$, for the
ideal-IRS scenario (without the presence of the PD) are shown in Fig. 3(a)-3(c), respectively, and those of the non-ideal-IRS scenario (with the presence of the PD) are shown in Fig. 3(d)- 
3(f), respectively. The AARs are displayed as increasing functions of $P_{S}$. Without the IRS, the received signal at each user propagates on the direct link and multi-path fading channel. Therefore, the AARs are very low, as shown as the lowest curves in Fig. 3. When the IRS is utilized, the AARs are significantly enhanced even with the presence of the PD. Although different levels of AAR enhancement are observed, these results still indicate the important role of the IRS in improving the RSSs at the users. As shown in Fig. 3(c) and 3(f), the total AARs for the cases of IRS[1,2] and IRS[2,1] (in these cases, the IRS assists the communication for both users) achieve better values than other cases.

In Fig. 4, we adopt the best IRS setup for the case of $P_{S}=0$ $\mathrm{dBm}$ with $\tau_{\mathrm{PD}}=0.1$ (found in Fig. 3, i.e., IRS[2,1]) and then investigate the effect of the PA factors on the total AAR. The advantages of the NOMA scheme are confirmed by investigating a reference orthogonal multiple access (OMA) scheme. Due to the advantageous location of the near user, the system achieves a highest or lowest total-AAR when allocating all resources to the near user or the far user, respectively. When the SIC imperfection factor $\zeta$ increases, the total AAR becomes worse. For a performance comparison, we use a conventional OMA scheme, which defines the AAR for $U_{m}, m=\{1,2\}$, as

$$
R_{m}^{\mathrm{OMA}}=v_{m} \log _{2}\left(1+\gamma_{m}\left|\hat{h}_{m}\right|^{2}\right),
$$

and the total AAR for OMA is given by

$$
\bar{C}_{\Sigma}^{\mathrm{OMA}}=\mathrm{E}\left\{R_{1}^{\mathrm{OMA}}\right\}+\mathrm{E}\left\{R_{2}^{\mathrm{OMA}}\right\} .
$$

where $v_{m} \in(0,1), v_{1}+v_{2}=1$, is the resource allocation (RA) factors for OMA (e.g., time allocation factor for TDMA or bandwidth allocation factor for FDMA). Compared to OMA,

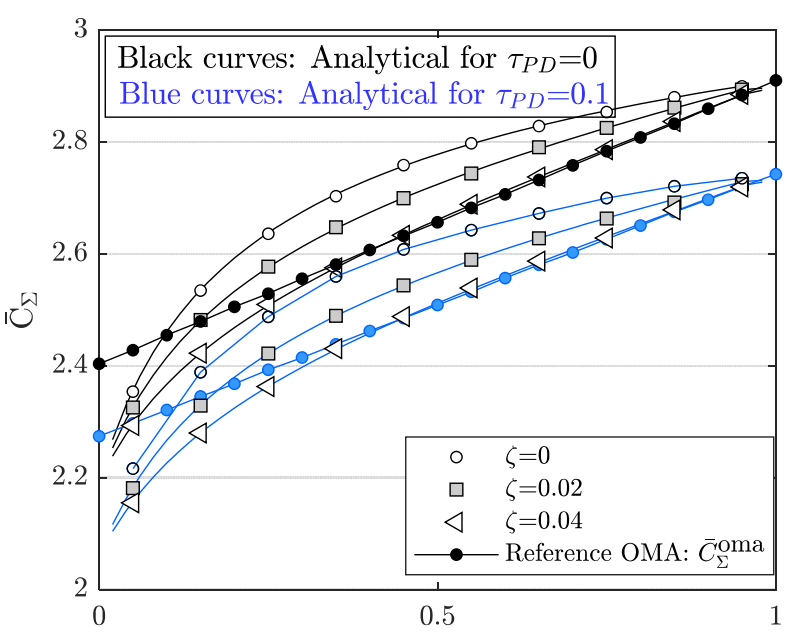

Fig. 4. The effects of PA factors for NOMA and RA factors for OMA on the system's capacities.
NOMA can achieve a higher total AAR in the presence of PD and imperfect-SIC. When $\zeta$ is sufficiently large, NOMA yields a lower performance compared to OMA. In Fig. 5, we show the joint effect of the RT-allocation strategy and PA factors, i.e., $\alpha_{1}$ and $\alpha_{2}$, on the AAR. Because the size of each RT is relatively small compared to the wave-propagation distances, the difference in distance between reflecting links is trivial. It is reasonable to assume that the signal attenuations of reflecting links toward a user are similar. For this reason, the RT allocation strategy is as follows. Let $N_{U_{1}}$ be the number of RTs allocated to $U_{1}$. These $N_{U_{1}}$ RTs are assigned from left to right and from top to bottom. The other $N_{U_{2}}=N-N_{U_{1}}$ RTs are assigned to $U_{2}$. For a given value of $N_{U_{1}}, \bar{C}_{1}$ increases, while $\bar{C}_{2}$ decreases when we increase $\alpha_{1}$ and vice versa. This is because the power resource allocated for the users is defined by $\alpha_{1}$. For given PA factors, $\bar{C}_{1}$ is shown as an increasing function of $N_{U_{1}}$, whereas $\bar{C}_{2}$ improves with the first increase in $N_{U_{1}}$ and then reaches the optimal value. Finally, it degrades with further increases in $N_{U_{1}}$. The trend of $\bar{C}_{2}$ is explained as follows. Since both users

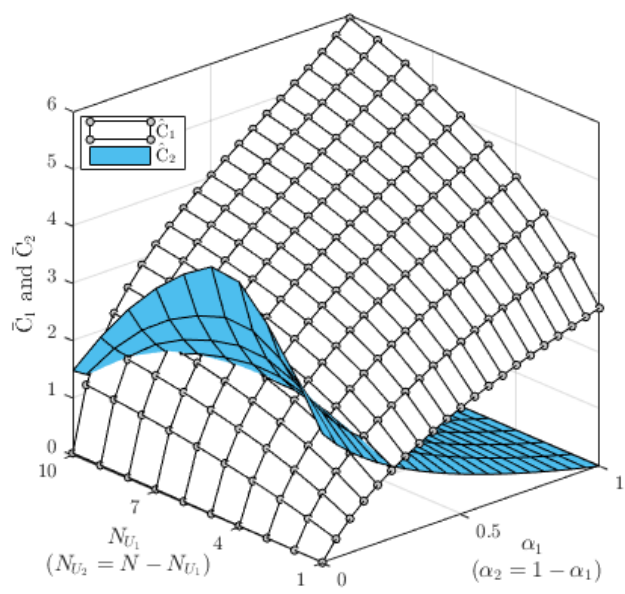

(a)

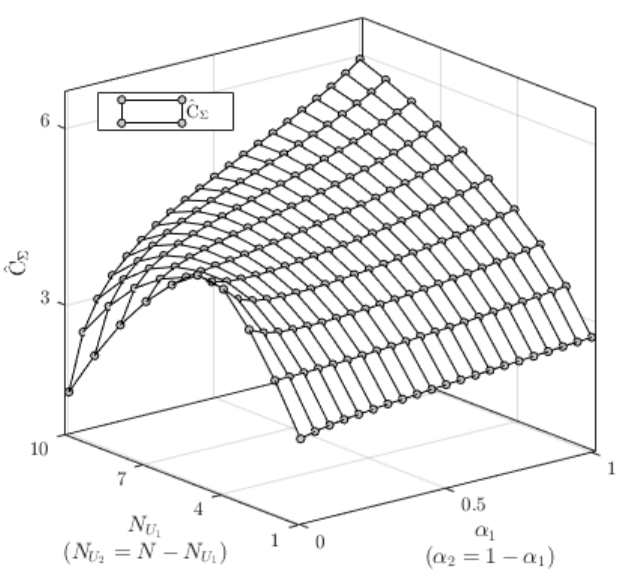

(b)

Fig. 5. The joint effect of PA factors and RT allocations on (a) the AARs for users and (b) the total AAR. 
need to decode $x_{2}$ successfully, the IRS needs assist both users to gain the highest value of $\bar{C}_{2}$. Therefore, using very high or very low values of $N_{U_{1}}$ does not yield good values for $\bar{C}_{2}$. Although the highest total AAR is observed at $\left(N_{U_{1}}=10, \alpha_{1}=\right.$ $1)$, the NOMA system does not use this configuration. The reason is that at this point, all system resources, including $P_{\mathrm{S}}$ and RTs, are allocated to $U_{1}$; hence, $U_{2}$ is disconnected from the network. For our proposed NOMA system, we focus on the points lying on the intersection curve of the two user-AAR surfaces denoted by $\ell\left(N_{U_{1}}, \alpha_{1}^{*}\right)$, on which two users can communicate with the source at the same AAR. Let the highest value observed on $\ell\left(N_{U_{1}}, \alpha_{1}^{*}\right)$ be the optimal NOMA-AAR $\ell^{*}=\max \ell\left(N_{U_{1}}, \alpha_{1}^{*}\right)$ and its respective coordinate $\left(N_{U_{1}}^{*}, \alpha_{1}^{*}\right)$ be the optimal solution for $\ell^{*}$. The trends of $\ell^{*}$ and $\left(N_{U_{1}}^{*}, \alpha_{1}^{*}\right)$ are studied in Fig. 6.

In Fig. 6, we consider an IRS with a size of 2-by- $N_{\text {col }}$ RTs and then plot the optimal NOMA-AAR as shown in Fig. 6(a) and its respective optimal solution, i.e., optimal PA factors as shown in Fig. 6(b) and optimal RT-allocation as shown in Fig.

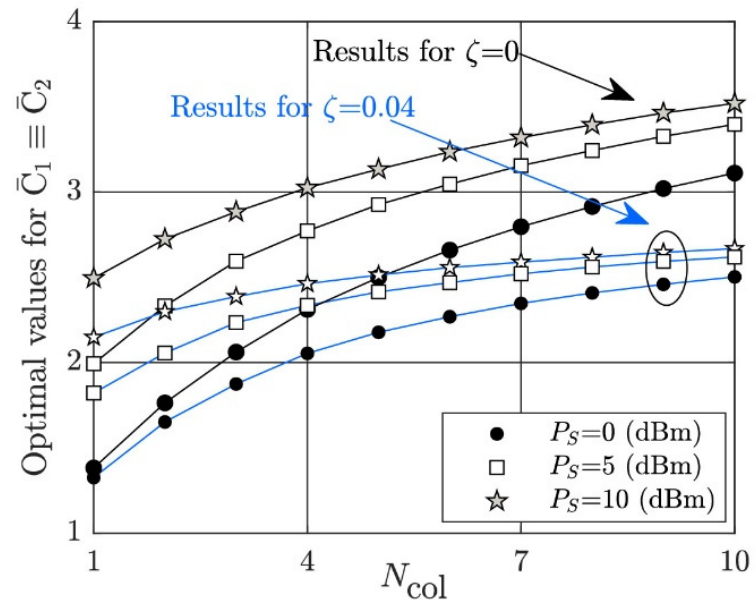

(a)

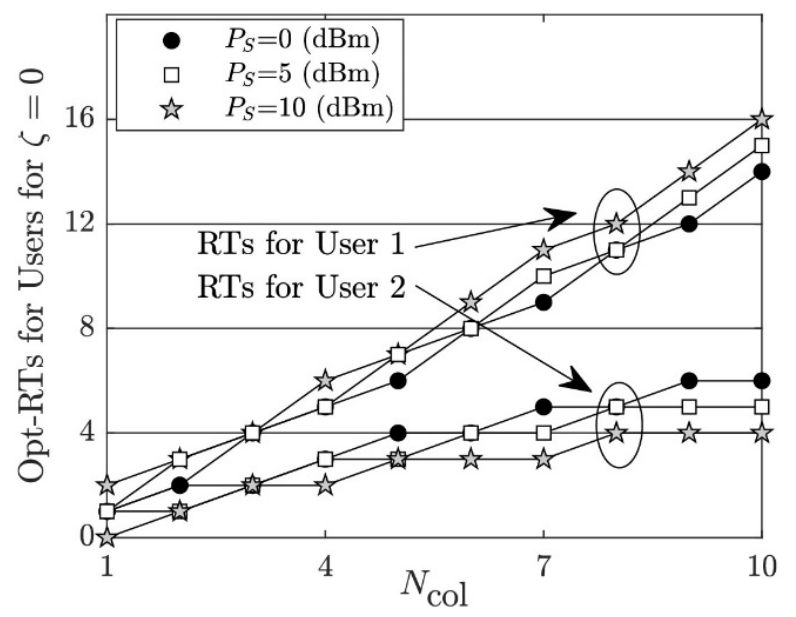

(c) 6(c) and 6(d), under different parameters of $P_{S}$ and $\zeta$. Fig. 6(a) shows that the more RTs are used, the higher value for optimal NOMA-AAR is achieved. When the IRS's size is sufficiently large, the increase in size does not gain much improvement in optimal NOMA-AAR. Therefore, the size of the IRS should be chosen carefully to achieve a reasonable trade-off between performance and cost. For instance, at $\zeta=0.04$ and $P_{S}=0$ $\mathrm{dBm}$, the 2-by- 1 and 2-by-4 IRSs can support $1 \mathrm{bit} / \mathrm{s} / \mathrm{Hz}$ and 2 bit/s/Hz optimal NOMA-AAR, respectively. In Fig. 6(b)-6(d), we show the trends of the obtained optimal solutions. Regarding the NOMA principle, first, both users need to decode the signal allocated with stronger power, which is $x_{2}$ for our proposed system, and consider the rest signals as interference, as shown in (12). After successfully decoding $x_{2}, U_{1}$ eliminates the signal $x_{2}$ from its received signal and then decodes its desired signal $x_{1}$, which is allocated with lower power. The allocated power for each signal is defined by the PA factors. Compared to $x_{2}$, the decoding process of $x_{1}$ deals with lower interference, which is caused by the imperfect-SIC decoder as shown

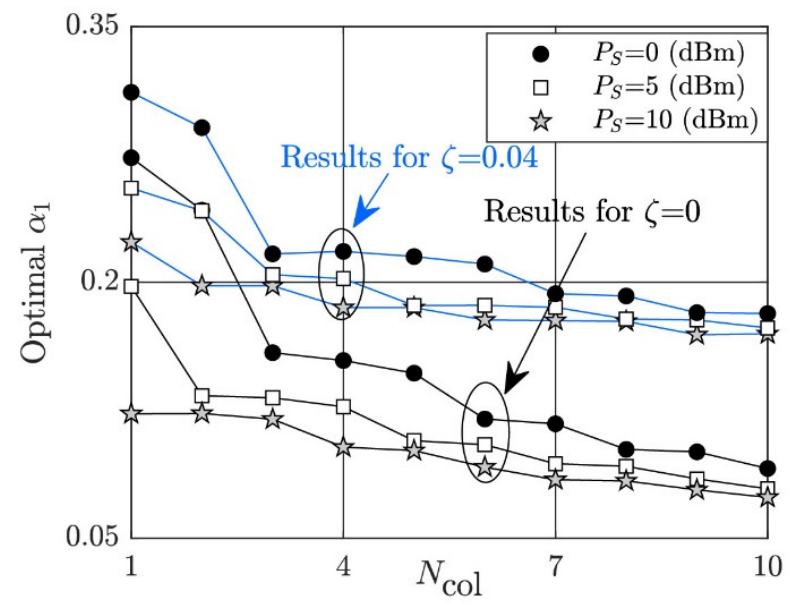

(b)

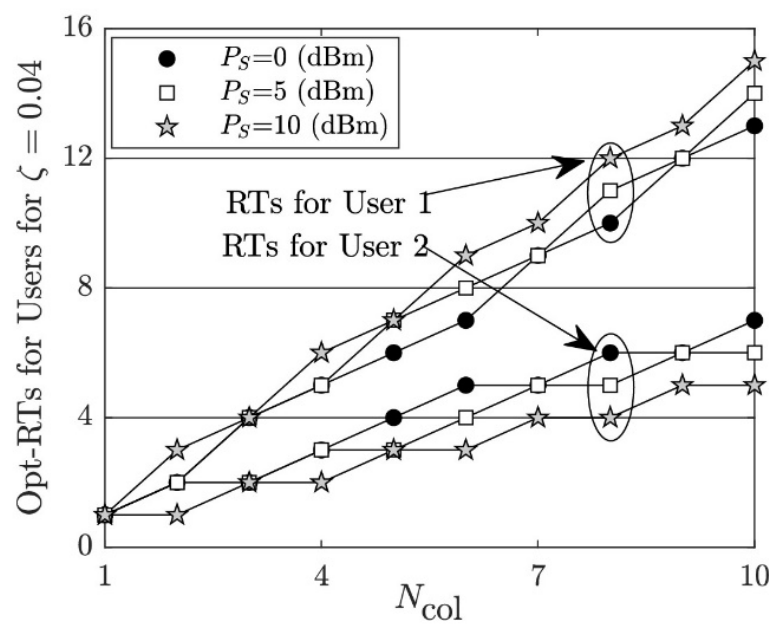

(d)

Fig. 6. The results of (a) optimal NOMA-AAR, (b) the optimal PA allocation factors, and (c, d) the optimal RT-allocation strategy. 
in (13). For this reason, the AAR for $x_{2}, \bar{C}_{2}$, significantly depends on the PA factors, and $\bar{C}_{2}$ increases when $\alpha_{1}^{*}$ decreases (or equivalently $\alpha_{2}^{*}$ increases). Moreover, $\bar{C}_{1}$ significantly depends on the RSS at $U_{1}$. Hence, $\bar{C}_{1}$ is enhanced when more RTs are allocated to $U_{1}$. Using the above assessments, it is easy to explain the trends of $\alpha_{1}^{*}$ and $N_{U_{1}}^{*}$. When the total number of RTs increases, the signal quality at users becomes greater, and the system can boost both AARs at two users by allocating more RTs to $U_{1}$ and using higher values for $\alpha_{2}^{*}$ (or equivalently using lower values for $\alpha_{1}^{*}$ ). Therefore, $\alpha_{1}^{*}$ is a decreasing function of $N_{\mathrm{col}}$, as shown in Fig. 6(b), whereas $N_{U_{1}}^{*}$ and $N_{U_{2}}^{*}$ are increasing functions of $N_{\mathrm{col}}$, as shown in Fig. 6(c) and 6(d). Moreover, $N_{U_{1}}^{*}$ increases more significantly than $N_{U_{2}}^{*}$. On the other hand, the increase in transmit power $P_{S}$ or the decrease in the SIC-imperfection factor $\zeta$ also give the same effect on the signal quality at the users.

Hence, the smaller value for $\alpha_{1}^{*}$ and higher values for $N_{U_{1}}^{*}$ are observed when $P_{S}$ increases or $\zeta$ decreases.

In Fig. 7, we show the effects of the PD factor $\tau_{\mathrm{PD}}$ and the

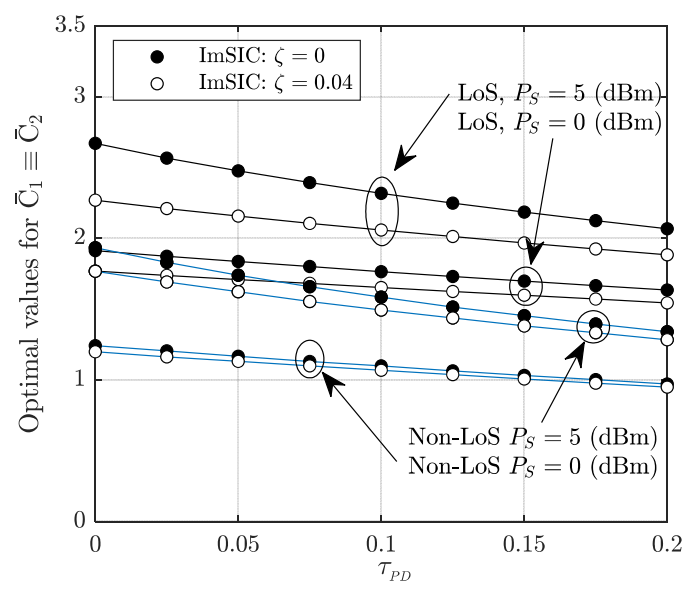

(a)

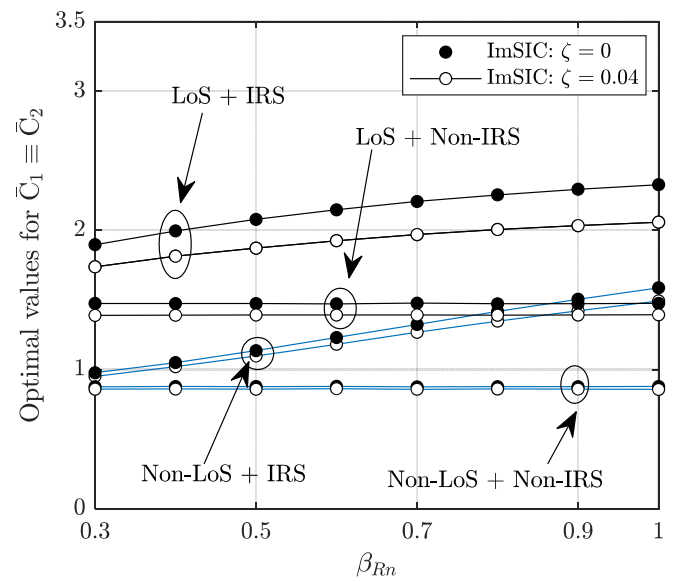

(b)

Fig. 7. (a) The effect of $P D$ and (b) the effect of reflection amplitude on the optimal NOMA-AAR. Two cases, with and without LoS links, are considered. Other parameters: $P_{s}=5$ dBm for Fig. 7(b). reflection amplitude $\beta_{R_{n}}$ on the system performance for two cases: with and without the presence of the LoS links. The results for the non-LoS case are obtained by set the source-to-user distances in (8) by very large values, i.e., $d_{S U_{m}} \rightarrow \infty$. The optimal NOMA-AAR drops around 0.8 bits $/ \mathrm{s} / \mathrm{Hz}$ when the $\mathrm{LoS}$ links are not available. This indicates the importance of the LoS links; however, without the LoS links, the system can achieve a moderately optimal NOMA-AAR with the help of the reflecting link and the multi-path fading channel. When $\tau_{\mathrm{PD}}$ increases, the total noise at each user increases. Hence, the optimal NOMA-AAR is displayed as a decreasing function of $\tau_{\mathrm{PD}}$ as shown in Fig. 7(a). With the presence of a multi-path fading channel and/or LoS links, the influence of the PD on the optimal NOMA-AAR can be reduced; however, when the user is blocked and its communication only relies on the reflecting links, the value of $\tau_{\mathrm{PD}}$ becomes a crucial factor to performance. Fig. 7(b) presents the optimal NOMA-AAR for different values of $\beta_{R_{n}}$. When $\beta_{R_{n}}$ increases, the stronger received signal strengths are achieved at the users. Therefore, the optimal NOMA-AAR is shown as an increasing function of $\beta_{R_{n}}$. Moreover, the optimal NOMA-AAR for the case using the IRS is higher than that for the non-IRS case. This indicates the effectiveness of the IRS in improving system performance. Since $\beta_{R_{n}}$ only affects the quality of the reflecting links, the influence of low $\beta_{R_{n}}$ values can be reduced in the case of the presence of the LoS links or the stronger multi-path fading channel.

\section{CONCLUSION}

This paper considered an IRS-aided NOMA communication in the presence imperfect SIC. The theoretical results are derived and confirmed by Monte Carlo simulations. The numerical results showed that the NOMA communication achieves a significant enhancement in performance with the help of an IRS, and it outperforms the reference OMA system. The residual interference caused by the imperfect SIC leads to a remarkable degradation in performance. There is an optimal setup for the PA factors and the number of RTs allocated to each user that allows the best equal capacity for both users. The effects of key system parameters on the trends of the optimal setup were studied. For instance, more RTs are allocated to the near user when the number of available RTs increases. Moreover, a higher portion of transmit power is allocated to the far user when the source transmit power increases.

This research was supported by Basic Science Research Program (No. 2020R1I1A3057142) and the Priority Research Centers Program (No. 2019R1A6A1A03032988) through the National Research Foundation of Korea. 


\section{REFERENCES}

[1] C. Ranaweera, E. Wong, A. Nirmalathas, C. Jayasundara, and C. Lim, "5G C-RAN with optical fronthaul: an analysis from a deployment perspective," Journal of Lightwave Technology, vol. 36, no. 11, pp. 2059-2068, 2018.

[2] H. Hashida, Y. Kawamoto, and N. Kato, "Intelligent reflecting surface placement optimization in air-ground communication networks toward 6G," IEEE Wireless Communications, vol. 27, no. 6, pp. 146-151, 2020.

[3] Y. Liu, Z. Qin, M. Elkashlan, Z. Ding, A. Nallanathan, and L. Hanzo, "Non-orthogonal multiple access for $5 \mathrm{G}$ and beyond," Proceedings of the IEEE, vol. 105, no. 12, pp. 23472381, 2017.

[4] C. Liaskos, S. Nie, A. Tsioliaridou, A. Pitsillides, S. Ioannidis, and I. Akyildiz, "A new wireless communication paradigm through software-controlled metasurfaces," IEEE Communications Magazine, vol. 56, no. 9, pp. 162-169, 2018.

[5] T. J. Cui, M. Q. Qi, X. Wan, J. Zhao, and Q. Cheng, "Coding metamaterials, digital metamaterials and programmable metamaterials," Light: Science \& Applications, vol. 3, article no. e218, 2014. https://doi.org/10.1038/1sa.2014.99

[6] Q. U. A. Nadeem, H. Alwazani, A. Kammoun, A. Chaaban, M. Debbah, and M. S. Alouini, "Intelligent reflecting surface-assisted multi-user MISO communication: channel estimation and beamforming design," IEEE Open Journal of the Communications Society, vol. 1, pp. 661-680, 2020.

[7] M. Najafi, V. Jamali, R. Schober, and H. V. Poor, "Physicsbased modeling and scalable optimization of large intelligent reflecting surfaces," IEEE Transactions on Communications, vol. 69, no. 4, pp. 2673-2691, 2020.

[8] O. Ozdogan, E. Björnson, and E. G. Larsson, "Intelligent reflecting surfaces: physics, propagation, and pathloss modeling," IEEE Wireless Communications Letters, vol. 9, no. 5, pp. 581-585, 2020.

[9] Y. Zheng, S. Bi, Y. J. Zhang, Z. Quan, and H. Wang, "Intelligent reflecting surface enhanced user cooperation in wireless powered communication networks," IEEE Wireless Communications Letters, vol. 9, no. 6, pp. 901-905, 2020.

[10] V. P. Tuan and I. P. Hong, "Secrecy performance analysis and optimization of intelligent reflecting surface-aided indoor wireless communications," IEEE Access, vol. 8, pp. 109440-109452, 2020.

[11] X. Ma, Z. Chen, W. Chen, Y. Chi, Z. Li, C. Han, and Q. Wen, "Intelligent reflecting surface enhanced indoor terahertz communication systems," Nano Communication Networks, vol. 24, article no. 100284, 2020. https://doi. org/10.1016/j.nancom.2020.100284

[12] J. Yuan, Y. C. Liang, J. Joung, G. Feng, and E. G. Larsson, "Intelligent reflecting surface-assisted cognitive radio system," IEEE Transactions on Communications, vol. 69, no. 1, pp. 675-687, 2020.

[13] H. Zhang, J. Hu, H. Zhang, B. Di, K. Bian, Z. Han, and L. Song, "Metaradar: indoor localization by reconfigurable metamaterials," IEEE Transactions on Mobile Computing, 2020. https://doi.org/10.1109/TMC.2020.3044603

[14] A. Papazafeiropoulos, B. Clerckx, and T. Ratnarajah, "Rate-splitting to mitigate residual transceiver hardware impairments in massive MIMO systems," IEEE Transactions on Vehicular Technology, vol. 66, no. 9, pp. 8196-8211, 2017.

[15] E. Costa and S. Pupolin, "M-QAM-OFDM system performance in the presence of a nonlinear amplifier and phase noise," IEEE Transactions on Communications, vol. 50, no. 3, pp. 462-472, 2002.

[16] T. Schenk, RF Imperfections in High-Rate Wireless Systems: Impact and Digital Compensation. Berlin, Germany: Springer-Verlag, 2008.

[17] E. Basar, M. Di Renzo, J. De Rosny, M. Debbah, M. S. Alouini, and R. Zhang, "Wireless communications through reconfigurable intelligent surfaces," IEEE Access, vol. 7, pp. 116753-116773, 2019.

[18] I. S. Gradshteyn, I. M. Ryzhik, A. Jeffrey, and D. Zwillinger, Table of Integral, Series and Products, 7th ed. Amsterdam, Netherlands: Elsevier, 2007.

[19] G. Zhu, C. Zhong, H. A. Suraweera, Z. Zhang, C. Yuen, and R. Yin, "Ergodic capacity comparison of different relay precoding schemes in dual-hop AF systems with cochannel interference," IEEE Transactions on Communications, vol. 62, no. 7, pp. 2314-2328, 2014.

[20] C. A. Balanis, Antenna Theory Analysis and Design, 2nd ed. New York, NY: John Wiley \& Sons, Inc., 1997. 


\section{Van Phu Tuan}

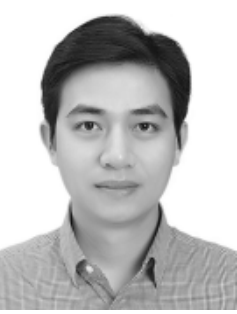

received B.E. and M.E. degrees in Electronics and Telecommunications Engineering from HoChiMinh City University of Technology, Vietnam, in 2010 and 2013, respectively, and a Ph.D. degree in Electrical Engineering from University of Ulsan, Korea, in 2018. From 2018 to 2019, he was a post-doctoral researcher at the University of Ulsan. Currently, he is working at Kongju National University, Korea. His major research interests are wireless communications systems, physical layer security, wireless powered communications, optimization methods, and reinforcement learning methods.

\section{Ic-Pyo Hong}

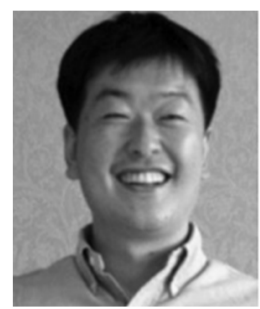

received B.S., M.S., and Ph.D. degrees in Electronics Engineering from Yonsei University, Seoul, South Korea, in 1994, 1996, and 2000, respectively. From 2000 to 2003, he was with the Information and Communication Division, Samsung Electronics Company, Suwon, South Korea, where he was a Senior Engineer with CDMA Mobile Research. Since 2003, he has been with the Department of Information and Communication Engineering, Kongju National University, Cheonan, South Korea, where he is currently a professor. In 2006 and 2012, he was a Visiting Scholar with Texas A\&M University, College Station, TX, USA, and Syracuse University, Syracuse, NY, USA, respectively. His research interests include numerical techniques in electromagnetics and periodic electromagnetic structures. 